\title{
Experiencing an Intimate Partner's Breast Cancer: Attachment, Caregiving, and Burden in Men
}

\author{
Marisa Ávila ${ }^{1}$, Tânia Brandão ${ }^{1}$, Joaquim Luís Coimbra ${ }^{1}$, Frederick Lopez ${ }^{2} \&$ Paula \\ Mena Matos ${ }^{1}$
}

${ }^{1}$ Faculty of Psychology and Education Sciences and the Centre for Psychology, University of Porto, Portugal.

${ }^{2}$ University of Houston

Address correspondence to Paula Mena Matos, Rua Alfredo Allen, 4200-135, Porto, Portugal. E-mail: pmmatos@fpce.up.pt

Funding: This study was supported by a doctoral grant (SFRH/BD/72730/2010) from the Portuguese Foundation for Science and Technology attributed to the first author

Please Note: This is the author's version of the manuscript accepted for publication in Psychiatry. Changes resulting from the publishing process, namely editorial editing, corrections, final formatting for publication, and other modifications resulting from quality control mechanisms may have been subsequently added.

A definitive version was subsequently published as: Ávila, M., Brandão, T., Coimbra, J.L., Lopez, F., \& Matos, P.M. (2016) Experiencing an Intimate Partner's Breast Cancer: Attachment, Caregiving, and Burden in Men, Psychiatry, 79, 3, 236-248, DOI:10.1080/00332747.2016.1158003 


\begin{abstract}
Objective: This study examined interrelationships among adult attachment orientations, caregiving, and caregiver burden in men of female partners with breast cancer, and tested whether caregiving patterns mediated associations between men's attachment orientations and their self-reported caregiver burden. Method: The participants were 124 male partners of women with breast cancer. These participants completed assessments related to attachment, caregiving, and caregiver burden. Path models examined the associations between constructs and tested mediational effects. Results: Findings demonstrated significant associations between men's adult attachment orientations and their experience of caregiver burden. In addition, the maintenance of proximity in caregiving completely mediated the respective associations of attachment security and attachment avoidance to caregiver health problems, on one hand, and to the caregiver's self-esteem (e.g., another indicator for caregiver burden), on the other. Moreover, we found a direct effect of attachment avoidance on health problems. Conclusions: This study highlighted the importance of addressing adult attachment dispositions and caregiving to understanding the relational processes implicated in caregiver burden. The results support the conclusion that men's adult attachment orientations and caregiving patterns toward their female partners with breast cancer are relevant contributors to men's perceptions of caregiver burden.
\end{abstract}

\title{
Introduction
}

The diagnosis of breast cancer instigates a major crisis not only for the patients but also for their primary caregivers. As primary caregivers, intimate partners may be especially overwhelmed by the practical and emotional demands of having to assume the caregiver role under sudden circumstances, with minimal preparation and uncertain support from the health care system (Glajchen, 2004), where their needs may be largely unmet (Adams et al., 2013). Moreover, caregivers' efforts to provide the appropriate type and level of support, to adjust to new family roles, and to regulate their personal feelings in relation to breast cancer might pose unique difficulties for them while providing care. It is wellknown that cancer caregivers experience difficulties that may compromise their own health and well-being (Braun, Mikulincer, Rydall, Walsh, \& Rodin, 2007; Girgis, Lambert, Johnson, Waller, \& Currow, 2013; Stenberg, Ruland, \& Miaskowski, 2010). Consequently, breast cancer studies have warned that caregivers' health may be affected negatively (Kim \& Given, 2008; Moreira \& Canavarro, 2013). Individuals who view their 
partner's breast cancer and its impact on their lives more negatively appear to be particularly vulnerable to burden. For example, they report higher levels of anxiety, depression, and physical problems (Pauwels, De Bourdeaudhuij, Charlier, Lechner, \& Van Hoof, 2012). In addition, there is evidence that quality of life (e.g., psychological well-being, social relationships, and physical health) of partners of patients with breast cancer was lower compared with partners of women without cancer (Moreira \& Canavarro, 2013). The latter finding suggests that intimate partners may become a second patient in need of care (Kim, Carver, Cannady, \& Shaffer, 2013; Li, Mak, \& Loke, 2012; Pitceathly \& Maguire, 2003). Given that breast cancer patients identify their partners as their preferential source of support (Falagas et al., 2007; Kayser, Watson, \& Andrade, 2007), it is important that research advances a comprehensive understanding of caregiver needs so that effective family support interventions can be developed. To date, however, research has mainly focused on the caregiver's negative experiences and outcomes (Li et al., 2012), and only a handful of studies have considered that caregiving might be associated with positive outcomes (i.e., positive meanings that arise from providing care to the partner, appreciating new relationships with others (Kim, Schulz, \& Carver, 2007; Manne et al., 2014), and experiencing greater closeness within the marital relationship (Dorval et al., 2005). Together these studies underscore the importance of conceptualizing caregiving burden as a multidimensional construct with important implications for developing models of breast cancer care. The present study therefore considers both negative and positive indicators of caregivers' well-being in its examination of caregiver burden. More specifically, because physical health has been shown to be an important issue for primary caregivers, we assessed the perception of physical health deterioration as a consequence of assisting

the partner with breast cancer. In addition, we assessed caregiver esteem, here conceptualized as the extent to which the caregiver either experiences feelings of enjoyment and reward or feelings of resentment when providing care (Given et al., 1992). Last, we drew on attachment theory (Bowlby, 1969) as a useful framework for organizing our inquiry and formulating our hypotheses regarding important interrelationships among men's adult attachment orientations, caregiving behaviors, and indicators of their caregiver burden. 
Attachment theory (Bowlby, 1969, 1977) is centrally concerned with how human beings develop patterns for regulating their affect in the context of their close, affectional relationships (i.e., attachments) and how these patterns influence their socioemotional functioning throughout the life span. Bowlby believed that these patterns were initially forged in the context of the infant-caregiver relationship and represented the operation of an innate motivational system (i.e., the attachment system). According to theory, when operating normatively the infant's experience of stress, discomfort, or uncertainty activates the attachment system and initiates care-seeking behaviors (e.g., crying). These behaviors would then be accurately discerned by the infant's caregiver, who would immediately provide the appropriate caregiving response (e.g., holding, feeding, comforting) for returning the infant's activated system to a quiescent state. Bowlby viewed the reliable repetition of these successful care-seeking/caregiving transactions as crucial to promoting the infant's autonomous functioning and affect regulation. Bowlby further argued that, within the first year of life, the infant would cognitively represent these transactions as part a favorable (i.e., secure) internal working model of close relationships. However, to the extent that an infant's caregivers were invasive, inconsistent, or rejecting of his or her care-seeking behaviors, the attachment system would itself become dysregulated, resulting in problematic transactional patterns that would later form the basis of an insecure secure internal working model. Last, because both secure and insecure working models possessed self-validating properties, they were assumed to respectively influence later adult relationships, as well as a wide range of adult behaviors, in favorable and unfavorable ways. Recently, Morse, Shaffer, Williamson, Dooley, and Schulz (2012) proposed that attachment theory could be used to conceptualize the relationship between caregivers and care recipients. According to these authors:

Bowlby (1982) argued that secure attachment is critical for responsive caregiving. Individuals give and seek support consistent with their models of self and other (Simpson, Rholes, \& Nelligan, 1992). The model of self describes whether people view themselves as worthy of care; model of others describes whether others can be trusted to provide care. A negative model of self is analogous to attachment anxiety; a negative model of others is analogous to attachment avoidance (Bartholomew \& Horowitz, 1991; Brennan, Clark, \& Shaver, 1998), Fraley, Waller, \& Brennan, 2000). (p. 211) 
Variability in how individuals provide care in their intimate adult relationships can thus be conceptualized as reflecting variability in their levels of anxiety about abandonment and avoidance of interpersonal closeness (Shaver \& Fraley, 2008). Furthermore, an attachment-related conceptualization of caregiving in intimate relationships is distinctive from caregiving in nonintimate relationships in that the former serves two major functions: to meet the partner's need for security (i.e., responding to signals of distress or potential threat) and to support the attached person's autonomy and exploration when such a person is not distressed (Collins, Ford, Guichard, \& Feeney, 2006). Adults with more secure attachment (i.e., those with low levels of both attachment anxiety and avoidance) showed a pattern of caregiving sensitivity and maintenance of proximity while meeting their partners' emotional needs. Individuals with a predominantly anxious attachment regularly engage in more compulsive and hypervigilant forms of caregiving (Braun et al., 2012; Kim \& Carver, 2007). Finally, adults with more avoidant attachment manifest a pattern of caregiving control expressed in terms of less proximity seeking and lower sensitivity toward their partner's needs for emotional reassurance and physical distance, which are the core of a self-reliant. pattern (Feeney \& Collins, 2003). Studies with couples have supported the association between adult attachment orientations and distinctive patterns of providing care in intimate relationships. Attachment security has been related to more frequent emotional care and positively linked to autonomous motives (e.g., altruistic or other oriented) and to experienced personal benefits from caregiving (Simpson, Winterheld, Rholes, \& Orina, 2007). In other studies, attachment security was an important predictor of caregivers' well-being and life satisfaction (Kim \& Carver, 2007; Kim, Carver, Deci, \& Kasser, 2008; Ybema, Kuijer, Hagedoorn, \& Buunk, 2002). By contrast, attachment anxiety has been linked to egoistic motives for caregiving, and attachment avoidance has been associated with greater difficulties in providing emotional care (Kim \& Carver, 2007). However, to our knowledge only one study analyzed attachment related implications to caregiving in the context of breast cancer. Askari, Madgaonkar, and Rowell (2012) found that relational variables were important predictors of depressive and anxiety symptoms in spouse caregivers. The study suggested that attachment security and marital satisfaction were protective factors preventing emotional problems (e.g., depression and anxiety). The study also advanced that the subjective perception of caregiver burden was a relevant predictor of depression and anxiety. In sum, theoretical expectations and empirical findings suggest that attachment differences are associated with different patterns of 
caregiving in intimate relationships. It is reasonable to assume that caregiving patterns will be associated with caregivers' perception of burden. Therefore, the study of caregiver burden might benefit from the recognition of the interplay between attachment and caregiving. Furthermore, the caregiving orientation (e.g., maintenance of proximity, caregiving control) might be the mechanism (e.g., mediational process) whereby attachment is associated with a positive or a negative perception of caregiver burden.

\section{THE PRESENT STUDY}

The present study explored the role of attachment and caregiving on caregiver burden among men whose intimate partners had been diagnosed with breast cancer. The following hypotheses were advanced: First, attachment security was expected to be associated with lower levels of caregiver burden (i.e., fewer health problems and higher caregiver esteem) whereas attachment insecurity (i.e., higher scores on both anxiety and avoidance dimensions) would be associated with higher caregiver burden (i.e., more health problems and lower caregiver esteem). Second, we expected that attachment security would be positively correlated with the caregiving patterns of maintenance of proximity and sensitivity. Attachment insecurity, on the other hand, was expected to be negatively associated with these same and positively associated with caregiving control. Finally, we expected that caregiving dimensions would mediate the anticipated relationships between attachment and caregiver burden. The conceptual model for the mediational hypothesis is represented in Figure 1.

\section{(INSERT FIGURE 1 AROUND HERE)}

\section{METHOD}

Participants and Procedures

Male partners of female breast cancer patients were recruited between 2011 and 2014 to participate in a broader study on couples' adaptations to breast cancer. Participant recruitment for this study, which received prior review and approval by the Ethical Committee of the Portuguese School of Oncological Studies, took place at two institutions: the Portuguese Institute of Oncology (Porto Breast Clinic) and the Portuguese Cancer League (Azores). The first institution is a public hospital and the second is a nonprofit organization that provides assistance for people dealing with cancer. Eligibility criteria required that participants be sufficiently fluent in Portuguese to 
complete self-report questionnaires. Detailed information regarding the study was given to all prospective participants, including an opportunity to clarify questions related with the study and concerning the roles of the researchers and the participants. Researchers approached the intimate male partners who accompanied patients to their clinical appointments at the Portuguese Institute of Oncology (Porto Breast Clinic) and the Portuguese Cancer League (Azores). Thus, this group of participants was approached directly in the clinical setting, and those who expressed interest in participating were immediately given a consent form to sign and the survey measures (described in the next section) to complete. However, the majority of the male partners (79\%) were recruited via patient referral, because women usually came alone or with other figures (e.g., friends and relatives) to receive treatment or to attend their clinical appointments. The researchers subsequently contacted these men by telephone to solicit their participation. Those who agreed to participate were sent a preaddressed envelope containing the informed consent form and the research measures, along with instructions to return these completed materials within 15 days. Subsequent to participant recruitment, the medical clinical registries were examined regarding clinical characteristics of the participants' partner and breast cancer patient. In all, 19 male participants were excluded from the study if their ailing partners were found to be (a) terminally ill, (b) with advanced breast cancer, or (c) exhibiting clinical comorbidities (e.g., psychiatric disorders). This resulted in a final sample of 124 men ranging in age from 27 to 77 years $(\mathrm{M}=52.74 ; \mathrm{SD}=9.08)$ and engaged in long-term relationships with their partners $(M=25.93$ years; $S D=10.22)$. Their female partners had been confronted with a clinical diagnosis of breast cancer in the past three years $(\mathrm{M}=2.95, \mathrm{SD}=4.90)$, and the majority of them were still undergoing treatment with chemotherapy $(n=76)$ and hormonotherapy $(n=15)$ at the time of the study.

Complete sociodemographic information on the final sample is presented in Table 1.

(INSERT TABLE 1 AROUND HERE)

\section{Measures}

Demographic Questionnaire

This measure gathered self-report information about participants' age, marital status, education, and monthly income. 


\section{Relationship Status}

Participants were asked to rate their intimate relationship according to their satisfaction through one question: "At the present moment how satisfied are you with your intimate relationship?" Participants rated this question with a Likert scale from Very unsatisfied (1) to Very satisfied (6). Caregivers reported high levels of satisfaction regarding their intimate relationships $(\mathrm{M}=5.29, \mathrm{SD}=1.14)$ at the moment of the study.

\section{Caregiver Burden}

Caregiver burden was assessed with the Caregiver Reaction Assessment (CRA) Scale (Given et al., 1992). The CRA is a 24-item questionnaire scored on a 5-point Likert-type scale designed to informally assess the caregiver's reactions. The CRA also consists of five subscales that quantify the caregiver's negative and positive experiences when providing care to a family member with physical and/or mental impairments (Given et al., 1992). For the purpose of this study, we only considered the health problems and caregiver esteem subscales. The health problems subscale (four items) consists of items concerning the caregiver's health and experience of vigor in relation to caregiving (e.g., "It takes all my physical strength to care for my partner"). Higher scores are indicative of more caregiver health problems. The caregiver esteem subscale (seven items) quantifies to what extent the caregiver experiences feelings of enjoyment and reward, or whether the situation arouses feelings of resentment (e.g., "Caring for my partner is important to me"). Higher scores on this subscale are indicative of higher caregiver esteem. In the present study, both subscales presented satisfactory internal consistency (Cronbach's alpha $=.71$ and .77 , respectively).

\section{Adult Attachment Orientations}

The short version of the Experiences in Close Relationships Scale (ECRS; Wei, Russell, Mallinckrodt, \& Vogel, 2007) was used to assess participants' adult attachment orientations. The ECRS is a 12-item questionnaire scored on a 7-point Likert-type scale that measures dimensions underlying adult attachment in terms of anxiety and avoidance. Anxiety refers to fear of rejection and abandonment (e.g., "I often worry about being abandoned"), and avoidance refers to discomfort with closeness in intimate relationships (e.g., "I try to avoid getting too close to my partner"). An alternative threefactor structure for this measure was used. based on prior assessment of the psychometric properties found in previous studies regarding to breast cancer (Ávila, 
Brandão, Teixeira, Coimbra, \& Matos, 2015). This alternative structure is also consistent with findings from other clinical studies (Braun et al., 2012). Cronbach's alpha in the present study was .91 for security (e.g., "I turn to my partner for many things, including comfort and reassurance," three items), .70 for anxiety (six items), and .75 for avoidance (three items).

\section{Caregiving}

The Caregiving Questionnaire (CQ; Kunce \& Shaver, 1994) was used to assess participants' patterns of caregiving in their intimate relationships. The 32-item CQ is composed of four subscales (i.e., maintenance of proximity, sensitivity, caregiving control, and compulsive caregiving) describing the different patterns of providing emotional and physical care in context to the couple's relationship. Given the aims of the present study, the following three CQ subscales were used: maintenance of proximity (eight items), which assesses an individual's ability to provide a distressed partner with physical and psychological accessibility (e.g., "When my partner is troubled or upset, I move closer to provide support and comfort"); sensitivity (eight items), which assesses the individual's ability to notice and accurately interpret a partner's needs, feelings, and nonverbal as well as verbal signals (e.g., "I can always tell when my partner needs comforting, even when she doesn't ask for it"); finally, control (eight items), which measures the extent to which the individual assists the partner's initiatives in regulating his or her personal needs (e.g., "When I try to help my partner; I sometimes end up 'taking over"'). In this study, Cronbach's alpha was .71 for maintenance of proximity, .73 for sensitivity, and .69 for control.

\section{Data Analyses}

As the total amount of missing data was less than 5\%, no cases with missing data were deleted, and expectation maximization (EM) was used to impute values for missing data based on an estimate the distribution of possible values for each missing data point (Schlomer, Bauman, \& Card, 2010). Mediational analyses were guided by the approach of Kenny and Judd (2010) meeting the standard assumptions of the general linear model. We anticipated that the relationship between attachment and caregiver burden could be explained through the mediating role of caregiving. Therefore, we estimated a saturated model in which the number of free parameters exactly equals the number of known values (i.e., a model with zero degrees of freedom). From this model, paths were sequentially 
dropped based on their significance and contribution to the overall model fit. This process of path examination was conducted for the final mediational model and further evaluated through bootstrapping analyses (using SPSS AMOS, Version 20.0, IBM Corp., Armonk, NY). Bootstrapping was chosen for being able to confirm or reject the mediational effects found in the model because of its accuracy for computing confidence intervals (MacKinnon, Fairchild, \& Fritz, 2007). Modification indices were then consulted to determine whether the fit of each model could be improved. We reported the standardized root mean square residual (SRMSR), root mean square error of approximation (RMSEA), comparative fit index (CFI), and model chi-square. Values below .05 for RMSEA, greater than .95 for CFI, and lower than .08 for SRMR indicated a good model fit (Hu \& Bentler, 1999). Bootstrapping analyses were then performed to examine the significance of the indirect effects.

\section{Results}

Table 2 presents the zero-order correlations as well as the means, standard deviations, and ranges of the scores for the study's key dimensions. Caregiver burden, adult attachment orientations, and caregiving patterns were intercorrelated in expected ways. Of note, participants' age was not significantly correlated with any of the study variables.

\section{(INSERT TABLE 2 AROUND HERE)}

Attachment, Caregiving, and Caregiver Burden: Tests of the Mediational Model

Figure 2 illustrates the outcome of the tests of the mediational model linking adult attachment orientations, caregiving patterns, and caregiver burden. These results demonstrated that the association between participants' attachment security and caregiver esteem was completely mediated by their reported capacity to maintain emotional proximity with their partners (maintenance of proximity: $\beta=.189, \mathrm{SE}=.047,95 \% \mathrm{CI}$ : lower bound $=.103$, upper bound $=.285, \mathrm{p}<.001)$. In addition, the link between participants' level of attachment avoidance and their caregiver esteem was completely explained by their self-reported difficulties in maintaining emotional proximity with their partners (maintenance. of proximity: $\beta=-.253, \mathrm{SE}=.065,95 \% \mathrm{CI}$ : lower bound $=-.384$, upper bound $=-.136, \mathrm{p}<.001)$. Moreover, we found a direct effect between participants' level of attachment avoidance and their self-reported health (health problems: $\beta=-.086$, 
$\mathrm{SE}=.032,95 \% \mathrm{CI}$ : lower bound $=.120$, upper bound $=.500, \mathrm{p}<.001)$. Finally, we found that none of the respective paths linking anxiety, sensitivity, and control to caregiver burden outcomes were significant; therefore, these paths were excluded from the final model, which offers a less complex and more parsimonious account of findings associated with the study's original hypotheses.

\section{DISCUSSION}

The present study examined the associations among adult attachment orientations, caregiving, and caregiver burden reported by a sample of intimate male partners of women with breast cancer. More specifically, it tested a mediational model wherein these caregivers' adult attachment orientations predicted their experience of caregiver burden directly and indirectly through their associations with caregiving patterns. Consistent with our expectations, we found that adult attachment orientations were related to participants' distinctive patterns of meeting their partners' emotional needs. Whereas more secure caregivers appeared altruistically motivated to help their relationship partners and manifested higher levels of proximity maintenance with them, more avoidant caregivers' reported lower levels of this caregiving pattern and thus acknowledged restricted emotional availability when providing physical and psychological comfort to their partners. These findings are consistent with prior studies in the breast cancer domain (Kim et al., 2008) and with studies using general population samples which have found that more avoidant individuals tend to dismiss their partners' expressed needs for support or care and instead tend to maintain little emotional involvement with them (Collins \& Feeney, 2000; Collins \& Ford, 2010; Feeney \& Collins, 2003). This pattern of emotional withdrawal from the partner's distress may protect the avoidant individual from dealing with the emotional upheaval associated with the activation of his own attachment system (Collins \& Feeney, 2000; Feeney \& Collins, 2003).

In addition, it is possible that caregivers' adult attachment characteristics may either buffer or intensify their experience of caregiver burden depending on whether these characteristics reflect secure or insecure attachment orientations. Specifically, more securely attached caregivers may experience their caring for the intimate partner as rewarding, which in turn may enhance their confidence and self-esteem as caregivers. In contrast, avoidantly attached caregivers may experience caregiving tasks as more emotionally challenging and taxing, which in turn may threaten their caregiver efficacy 
and self-perceptions as self-reliant individuals. Moreover, attachment avoidance exhibited a direct effect on health problems. Indeed, research has generally supported that insecure attachment is a risk factor for the development of adverse health conditions, in particular those involving the cardiovascular system and chronic diseases (Feeney, 2001; Maunder \& Hunter, 2008). Contrary to our expectations, anxiety did not predict maintenance of proximity. Considering the couple as an emotional system, we can preview that more anxious individuals may be more vulnerable to partner distress and find difficulty in differentiating their partners' distress from their own. There may be some form of attachment anxiety "contagion" occurring in couples, whereby having an anxious partner may be stressful and requires an added support provision, which may not be well received and may increase one's own fears (Davila \& Kashy, 2009). Future studies should therefore address the interplay between attachment and caregiving of both partners. In addition, it is possible that the gender of the caregiver needs to be included in future tests of models linking adult attachment, caregiving, and caregiver burden. Research suggests that differences in attachment, namely with regard to attachment anxiety, may be associated with sex differences (Del Giudice \& Belsky, 2010; Del Giudice, 2011). However, high anxiety may not always be linked to maintenance of proximity in every situation, especially in situations where individuals perceive that their partners are more stressed. In addition, we must consider that breast cancer constitutes a major stress and may trigger higher attachment anxiety resulting from the perceived changes that threaten the individual's emotional balance. Changes at the level of attachment anxiety may not be straightforwardly linked to negative outcomes, as they may reflect the organic and dynamic variation resulting from the activation of the attachment system. Nevertheless, further research is necessary for assessing differences in caregivers' perception of burden based on degree of anxiety, as its effects on burden might not be straightforwardly linked to a negative outcome. We found that caregivers' self-reported capacity to maintain emotional proximity with their partners constitutes an important mediating variable in explaining the association between adult attachment orientations and experiences of caregiver burden. Maintenance of proximity describes a pattern of secure emotional and physical availability toward the partner. Because breast cancer is a potentially life-threatening illness, it presents a clear threat to the attachment bond, as it suggests that the person diagnosed with cancer is less available as an attachment figure and may abandon their partner through role changes (i.e., hospitalization, disability). 
Because of this threat, the caregiver may seek proximity or closeness to his attachment figure as a response to the emotional distress he experiences due to his partner's breast cancer diagnosis and treatment. Gaining proximity may decrease the feeling of threat in the caregiver by increasing the sense of security and comfort through physical and emotional closeness (Burwell, Brucker, \& Shields, 2006). Indeed, attachment-oriented psychological interventions have been proven to be effective in optimizing breast cancer patients' and their partners' coping by promoting maintenance of proximity (Nicolaisen et al., 2014). This finding underscores the power of secure attachment bonds to promote health and well-being as well as general resilience, particularly in the face of traumatic events (Naaman, Radwan, \& Johnson, 2009).

\section{Limitations and Contributions for Research}

Although the present investigation adds to the meager literature extending attachment theory to research on the caregiving and caregiver burden reported by male partners of women with breast cancer, our findings must be considered alongside several study limitations. First, we employed a cross-sectional design, which precludes forming clear cause-and-effect inferences about interrelationships among our key study variables. Moreover, we limited our analyses to the attachment characteristics and caregiving experiences of the male partners of women diagnosed with breast cancer and did not consider the attachment characteristics and care-receiving experiences of these women. Longitudinal studies involving both members of a couple are necessary to more clearly test these inferences and to identify caregiver/care recipient relational patterns associated with both adaptive and problematic trajectories.

In addition, we exclusively relied on self-reported measures of these variables, which may have been vulnerable to social desirability bias and which may have conflated variable relationships due to common-method variance. Thus, multi-informant approaches to data collection involving evaluation of both members of the couple combined with more sophisticated methods of analysis, such as actor-partner interdependence models, are recommended for further studies. Despite these limitations, our study findings highlight the importance of assessing the attachment dispositions and emotional needs of the intimate partner caregivers of women with breast cancer when designing interventions to support these couples. Attachment-based interventions for coping with breast cancer may prove useful in improving each partner's abilities to communicate needs, express positive 
and negative feelings, and work out means of supporting one another, thus promoting adaptive caregiving and reducing caregiver burden. Future studies should focus on developing and evaluating the efficacy of couple attachment-based interventions in context to breast cancer. 